\title{
Current status of luminosity measurement with the CMD-3 detector at the VEPP-2000 collider
}

\author{
A.E. Ryzhenenkov ${ }^{1,2, *}$, R.R. Akhmetshin ${ }^{1}$, A.N. Amirkhanov ${ }^{1,2}$, A.V. Anisenkov ${ }^{1,2}$, V.M. \\ Aulchenko $^{1,2}$, V.Sh. Banzarov ${ }^{1}$, N.S. Bashtovoy ${ }^{1}$, D.E. Berkaev ${ }^{1,2}$, A.E. Bondar ${ }^{1,2}$, A.V. \\ Bragin $^{1}$, S.I. Eidelman ${ }^{1,2,5}$, D.A. Epifanov ${ }^{1,2}$, L.B. Epshteyn ${ }^{1,2,3}$, A.L. Erofeev ${ }^{1,2}$, G.V. \\ Fedotovich $^{1,2}$, S.E. Gayazov ${ }^{1,2}$, A.A. Grebenuk ${ }^{1,2}$, S.S. Gribanov ${ }^{1,2}$, D.N. Grigoriev ${ }^{1,2,3}$, F.V. \\ Ignatov $^{1}$, V.L. Ivanov ${ }^{1,2}$, S.V. Karpov ${ }^{1}$, V.F. Kazanin ${ }^{1,2}$, I.A. Koop ${ }^{1,2}$, A.A. Korobov ${ }^{1,2}$, \\ A.N. Kozyrev ${ }^{1,3}$, E.A. Kozyrev ${ }^{1,2}$, P.P. Krokovny ${ }^{1,2}$, A.E. Kuzmenko ${ }^{1}$, A.S. Kuzmin ${ }^{1,2}$, \\ I.B. Logashenko ${ }^{1,2}$, P.A. Lukin ${ }^{1,2}$, K.Yu. Mikhailov ${ }^{1}$, V.S. Okhapkin ${ }^{1}$, A.V. Otboev ${ }^{1}$, E.A. \\ Perevedentsev $^{1,2}$, Yu.N. Pestov ${ }^{1}$, A.S. Popov ${ }^{1,2}$, G.P. Razuvaev ${ }^{1,2}$, A.A. Ruban ${ }^{1}$, N.M. \\ Ryskulov $^{1}$, Yu.M. Shatunov ${ }^{1}$, P.Yu. Shatunov ${ }^{1}$, V.E. Shebalinn ${ }^{1,2}$, D.N. Shemyakin ${ }^{1,2}$, B.A. \\ Shwartz $^{1,2}$, D.B. Shwartz ${ }^{1,2}$, A.L. Sibidanov ${ }^{1,4}$, E.P. Solodov ${ }^{1,2}$, A.A. Talyshev ${ }^{1,2}$, S.S. \\ Tolmachev $^{1}$, A.I. Vorobiov ${ }^{1,2}$, and Yu.V. Yudin ${ }^{1,2}$ \\ ${ }^{1}$ Budker Institute of Nuclear Physics, SB RAS, Novosibirsk, 630090, Russia \\ ${ }^{2}$ Novosibirsk State University, Novosibirsk, 630090, Russia \\ ${ }^{3}$ Novosibirsk State Technical University, Novosibirsk, 630092, Russia \\ ${ }^{4}$ University of Victoria, Victoria, BC, Canada V8W 3P6 \\ ${ }^{5}$ Lebedev Physical Institute RAS, Moscow, 119333, Russia
}

\begin{abstract}
The CMD-3 detector started data taking at the electron-positron collider VEPP-2000 [1] in December 2010. The collected data sample corresponds to an integrated luminosity of $87 \mathrm{pb}^{-1}$ in the c.m. energy range from 1.06 up to $2 \mathrm{GeV}$ and $91 \mathrm{pb}^{-1}$ from 0.32 to $1.06 \mathrm{GeV}$. The integrated luminosity was measured by counting $e^{+} e^{-} \rightarrow e^{+} e^{-}$and $e^{+} e^{-} \rightarrow \gamma \gamma$ events, allowing additional photons in the final state. Preliminary results of the luminosity measurement are presented for various energy ranges and its accuracy is estimated to be $1 \%$.
\end{abstract}

\section{Introduction}

Precise luminosity measurement is a key requirement for many experiments studying hadronic cross sections at $e^{+} e^{-}$colliders. As a rule, a systematic uncertainty of the luminosity determination is one of the largest sources of the total uncertainty, which can significantly reduce the hadronic cross section accuracy. Therefore it is very important to determine the luminosity by using several well-known QED processes such as $e^{+} e^{-} \rightarrow e^{+} e^{-}, \mu^{+} \mu^{-}, \gamma \gamma$. Their combined usage will help to better understand and estimate the systematic accuracy of the luminosity measurement. The CLEO collaboration was the first to show in practice how a combined usage of the processes $e^{+} e^{-} \rightarrow e^{+} e^{-}, \mu^{+} \mu^{-}$and $\gamma \gamma$ helped to achieve a $1 \%$ accuracy for luminosity measurement [2].

The process $e^{+} e^{-} \rightarrow \gamma \gamma$ has essential advantages for luminosity determination [3, 4] compared to the first two. This process is free of effects due to radiation of the final-state particles

*e-mail: tem_mc@mail.ru 
Table 1. Integrated luminosity collected by CMD-3

\begin{tabular}{lll}
\hline Run & c.m. energy, GeV & Luminosity, $\mathrm{pb}^{-1}$ \\
\hline 2011 & $1.0-2.0$ & 20 \\
2012 & $1.2-2.0$ & 14 \\
2013 & $0.32-1.06$ & 24 \\
2017 & $1.2-2.0$ & 53 \\
2018 & $0.55-1.06$ & 67 \\
\hline
\end{tabular}

and Coulomb interaction. These reasons are the main motivation to explore this process as an independent tool for luminosity determination. Preliminary results of the luminosity determination are presented in a wide energy range.

\section{CMD-3 detector and dataset}

The Cryogenic Magnetic Detector [5], CMD-3, is a general-purpose detector. The cylindrical drift chamber (DC) measures the coordinates, angles and momenta of charged particles. The resolution along the beam axis is $\sim 2 \mathrm{~mm}$ as measured by charge division along the wires. The proportional Z-chamber mounted outside the DC provides a more accurate Z-coordinate measurement of the tracks. The resulting z-coordinate resolution is $\sim 500 \mu \mathrm{m}$. The calorimeter consists of three subsystems. The endcap BGO calorimeter with a depth of $13.4 X_{0}$ is placed on both sides of the DC flanges. The barrel part, which is placed outside the superconducting solenoid with a $1.3 \mathrm{~T}$ magnetic field $\left(0.13 X_{0}\right)$, consists of two systems: an inner Liquid Xenon calorimeter $\left(5.4 X_{0}\right)$ and a calorimeter based on CsI crystals with a depth of $8.1 X_{0}$. The analog strip information from the LXe calorimeter allows one to measure coordinates of the photon conversion point with a precision of about 1-2 $\mathrm{mm}$. The detector is surrounded by the muon range system.

In 2011 and 2012, $34 \mathrm{pb}^{-1}$ of integrated luminosity were collected in the c.m. energy range from $1.0 \mathrm{GeV}$ to $2.0 \mathrm{GeV}$. In 2013 the energy range from $0.32 \mathrm{GeV}$ to $1 \mathrm{GeV}$ was scanned with $10 \mathrm{MeV}$ steps. Integrated luminosity of $24 \mathrm{pb}^{-1}$ was collected in 2013. Since 2017 operation went on with the upgraded injection facility that allowed one an increase of the average luminosity by a factor of three. The 2017 scan was carried out in the energy range from 1.2 to $2.006 \mathrm{GeV}$ and the collected luminosity was $53 \mathrm{pb}^{-1}$. The 2018 season was dedicated to the energy scan below $1.06 \mathrm{GeV}$, and about $67 \mathrm{pb}^{-1}$ was collected in this run.

The beam energy measurement system [6] has been installed and commissioned in 2012. The system allows one to continuously monitor the beam energy with a relative precision better than $10^{-4}$, using Compton backscattering of laser photons by the electron beam.

\section{Luminosity measurement}

The integrated luminosity was measured using two well-known QED processes $e^{+} e^{-} \rightarrow e^{+} e^{-}$ and $e^{+} e^{-} \rightarrow \gamma \gamma$ according to the following formula:

$$
\int L \cdot \mathrm{d} t=\frac{N}{\sigma_{\text {Born }} \cdot \epsilon_{\text {rad }} \cdot \epsilon}
$$

where $N$ is the number of selected Bhabha or $\gamma \gamma$ events, $\sigma_{\text {Born }}$ is the Born cross section integrated within the detector acceptance, $\epsilon_{\text {rad }} \sim 0.947 \pm 0.002$ is the radiative correction calculated using the MCGPJ [7] generator and $\epsilon$ is the event detection efficiency. 


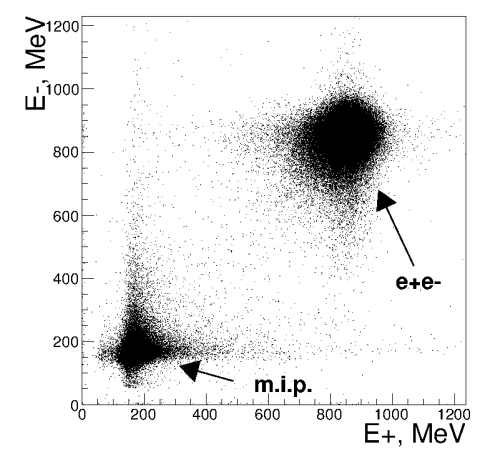

Figure 1. Correlation between energy depositions in calorimeters for two collinear tracks.

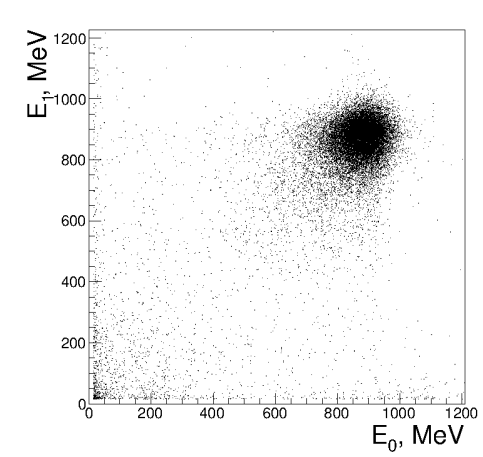

Figure 2. Correlation between energy depositions in calorimeters for two collinear photons.

Samples of collinear events $e^{+} e^{-}, \mu^{+} \mu^{-}, \pi^{+} \pi^{-}, K^{+} K^{-}$and cosmic background were selected for the luminosity determination. A correlation between energy depositions in calorimeters for these events is presented in Fig. 1 for the beam energy of $950 \mathrm{MeV}$. It is clearly seen that Bhabha events are located predominantly in the upper right corner, whereas other particles are populating the bottom left corner. A two-dimensional plot of the $e^{+} e^{-} \rightarrow \gamma \gamma$ energy deposition $E_{o}$ vs $E_{1}$ is presented in Fig. 2.

The detection efficiency of $e^{+} e^{-} \rightarrow e^{+} e^{-}$events is determined as $\epsilon=\epsilon_{2 \mathrm{tr}} \cdot \epsilon_{\mathrm{trg}} \cdot \epsilon_{\mathrm{cal}} \cdot \epsilon_{e n}^{2}$, where $\epsilon_{2 t r}$ is the track reconstruction efficiency in the DC, $\epsilon_{t r g}$ is the trigger efficiency, $\epsilon_{c a l}$ is the cluster reconstruction efficiency, $\epsilon_{e n}$ is the cluster selection efficiency for the energy depositions in the calorimeters.

The detection efficiency of the $e^{+} e^{-} \rightarrow \gamma \gamma$ events is determined as $\epsilon=\epsilon_{c a l} \cdot \epsilon_{e n}^{2} \cdot \epsilon_{n t r}$, where $\epsilon_{c a l}$ is the cluster reconstruction efficiency, $\epsilon_{e n}$ is the cluster selection efficiency for the energy deposition in the calorimeters, $\epsilon_{n t r}$ is the neutral trigger efficiency.

\section{Systematic uncertainties}

The fiducial volume of the CMD-3 detector can be determined independently with the LXe calorimeter and Z-chamber. It allows one to monitor the detector operation stability during data collection. The possibility of cross-checking a z-scale measurement with two subsystems allows us to control the systematic uncertainty from this source at a level of $0.1 \%$, and current conservative estimation is at a level of $0.3 \%$.

Another important source of systematic uncertainty is the theoretical precision of radiative corrections [8]. Additional studies are required in this field and comparison with experimental data is necessary. We expect that this uncertainty can be reduced to $0.1 \%$.

Event selection criteria for collinear tracks as well as some other factors also contribute to the systematic uncertainty of the integrated luminosity.

\section{Conclusion}

The VEPP-2000 collider successfully operates with a goal of collecting $\sim 1 \mathrm{fb}^{-1}$ in ten years and provide new precise results on hadron physics. The collected data correspond to an integrated luminosity of $178 \mathrm{pb}^{-1}$. The already collected data sample has the same or better statistical precision for the hadronic cross sections than achieved in previous experiments. 


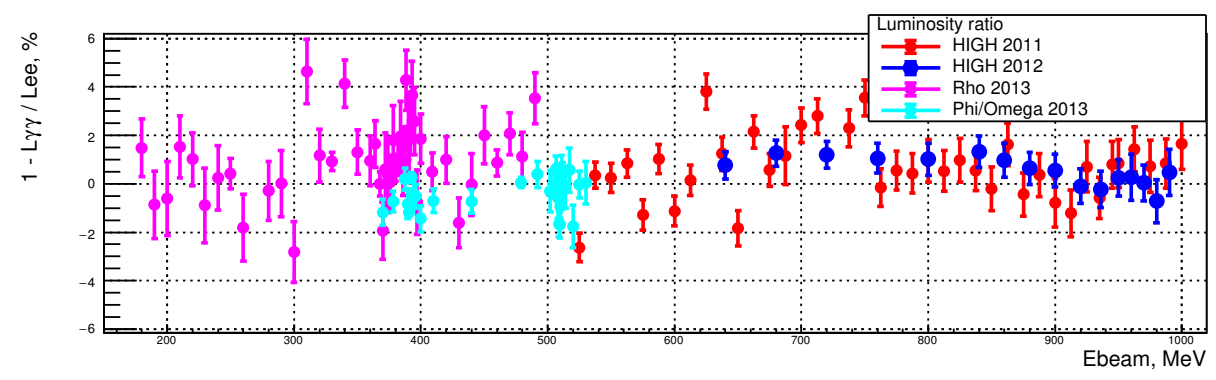

Figure 3. The ratio of the luminosities for the processes $e^{+} e^{-}$and $\gamma \gamma$ as a function of energy. Data are shown for the runs of 2011-2013.

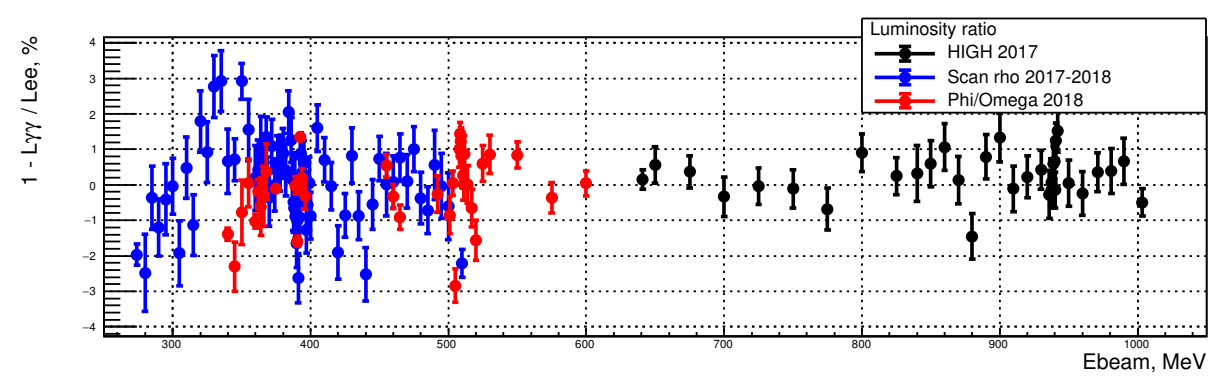

Figure 4. The ratio of the luminosities for the processes $e^{+} e^{-}$and $\gamma \gamma$ as a function of energy. Preliminary results are shown for the runs of 2017-2018.

The current integrated luminosity of the collider was measured using two well-known QED processes $e^{+} e^{-} \rightarrow e^{+} e^{-}$and $\gamma \gamma$. The ratio of the luminosities from the two processes as a function of energy is shown in Fig. 3 and 4, where only statistial uncertainties are shown. The current luminosity accuracy is estimated to be $1 \%$. The study of the different systematics is still in progress. In the close future we hope to reduce it to a $\sim 0.5 \%$ level.

\section{Acknowledgements}

We thank the VEPP-2000 personnel for excellent machine operation. The work was supported by the Russian Foundation for Basic Research grants RFBR 17-02-00847-a and RFBR 17-02-00897-a.

\section{References}

[1] I. Koop et al., Nucl. Phys. B, Proc. Suppl. 181, 371 (2008).

[2] G. Grawford et al., NIM A345, 429 (1994).

[3] E. Bartos et al., JETP Lett. 87, 73 (2008).

[4] S. Eidelman et al., EPJ C71, 1597 (2011).

[5] B. I. Khazin et al., Nucl. Phys. B, Proc. Suppl. 181-182, 376 (2008).

[6] E. V. Abakumova et al., Phys. Rev. Lett. 110, 140402 (2013).

[7] A. Arbuzov et al., EPJ C46, 689 (2006).

[8] S. Actis et al., Eur. Phys. J. C66, 585 (2010). 